\title{
BMJ Open Sleeve gastrectomy versus Roux-en-Y gastric bypass for type 2 diabetes and morbid obesity: double-blind randomised clinical trial protocol
}

\author{
Rinki Murphy, ${ }^{1}$ Nicholas J Evennett, ${ }^{2}$ Michael G Clarke, ${ }^{2}$ Steven J Robinson, ${ }^{2}$ \\ Lee Humphreys, ${ }^{2}$ Bronwen Jones, ${ }^{2}$ David D Kim, ${ }^{3}$ Richard Cutfield, ${ }^{3}$ \\ Lindsay D Plank, ${ }^{4}$ Hisham Hammodat, ${ }^{2}$ Michael W C Booth ${ }^{2}$
}

To cite: Murphy R,

Evennett NJ, Clarke MG, et al. Sleeve gastrectomy versus Roux-en-Y gastric bypass for type 2 diabetes and morbid obesity: double-blind randomised clinical trial protocol. BMJ Open 2016;6 e011416. doi:10.1136/ bmjopen-2016-011416

- Prepublication history for this paper is available online. To view these files please visit the journal online (http://dx.doi.org/10.1136/ bmjopen-2016-011416).

Received 5 February 2016 Revised 13 May 2016 Accepted 7 June 2016

CrossMark

For numbered affiliations see end of article.

Correspondence to

Dr Rinki Murphy;

R.Murphy@auckland.ac.nz

\section{ABSTRACT}

Introduction: Type 2 diabetes (T2D) in association with obesity is an increasing disease burden. Bariatric surgery is the only effective therapy for achieving remission of T2D among those with morbid obesity. It is unclear which of the two most commonly performed types of bariatric surgery, laparoscopic sleeve gastrectomy (LSG) and laparoscopic Roux-en-Y gastric bypass (LRYGB), is most effective for obese patients with T2D. The primary objective of this study is to determine whether LSG or LRYGB is more effective in achieving $\mathrm{HbA}_{1 \mathrm{c}}<6 \%$ ( $\left.<42 \mathrm{mmol} / \mathrm{mol}\right)$ without the use of diabetes medication at 5 years.

Methods and analysis: Single-centre, double-blind (assessor and patient), parallel, randomised clinical trial (RCT) conducted in New Zealand, targeting 106 patients. Eligibility criteria include age 20-55 years, T2D of at least 6 months duration and body mass index $35-65 \mathrm{~kg} / \mathrm{m}^{2}$ for at least 5 years. Randomisation $1: 1$ to LSG or LRYGB, used random number codes disclosed to the operating surgeon after induction of anaesthesia. A standard medication adjustment schedule will be used during postoperative metabolic assessments. Secondary outcomes include proportions achieving $\mathrm{HbA}_{1 \mathrm{c}}<5.7 \%$ (39 mmol/mol) or $\mathrm{HbA}_{1 \mathrm{c}}<6.5 \%$ ( $48 \mathrm{mmol} / \mathrm{mol}$ ) without the use of diabetes medication, comparative weight loss, obesity-related comorbidity, operative complications, revision rate, mortality, quality of life, anxiety and depression scores. Exploratory outcomes include changes in satiety, gut hormone and gut microbiota to gain underlying mechanistic insights into $\mathrm{T} 2 \mathrm{D}$ remission.

Ethics and dissemination: Ethics approval was obtained from the New Zealand regional ethics committee (NZ93405) who also provided independent safety monitoring of the trial. Study commenced in September 2011. Recruitment completed in October 2014. Data collection is ongoing. Results will be reported in manuscripts submitted to peer-reviewed journals and in presentations at national and international meetings.

Trial registration numbers: ACTRN12611000751976, NCT01486680; Pre-results.

\section{Strengths and limitations of this study}

- There is limited evidence from randomised clinical trials comparing the efficacy of laparoscopic sleeve gastrectomy (LSG) versus laparoscopic Roux-en-Y gastric bypass (LRYGB) to guide optimum surgery selection for morbidly obese patients with type 2 diabetes (T2D)

- We describe our double-blind, randomised trial designed to compare efficacy of LSG and silastic ring $L R Y G B$ on remission of T2D at 5 years among morbidly obese patients. We used a standard metabolic medication adjustment protocol after surgery, which should assist clinicians managing patients following bariatric surgery and researchers planning future bariatric surgery trials, given that the thresholds for discontinuing and restarting blood pressure, glucose and lipid medications postoperatively are frequently not reported.

- Limitations include the single-centre study design, which may limit generalisability of the findings.

\section{INTRODUCTION}

It is unclear which of the two major types of bariatric surgery, laparoscopic sleeve gastrectomy (LSG) and laparoscopic Roux-en-Y gastric bypass (LRYGB), achieves the greatest and most durable remission of type 2 diabetes (T2D) and weight loss. ${ }^{1}{ }^{2}$ There are currently only two prospective, non-blinded, randomised clinical trials (RCTs) comparing these two types of bariatric surgery ${ }^{4}$ in patients with T2D and one blinded study comparing the 'mini'-(one anastomosis) gastric bypass with LSG. ${ }^{5}$ In one study of 150 American patients with T2D (body mass index (BMI) $27-43 \mathrm{~kg} / \mathrm{m}^{2}$ ) randomised to LRYGB, LSG or medical therapy, $42 \%$ after LRYGB, 37\% after LSG and 12\% after 
medical therapy achieved diabetes remission at 12 months defined by $\mathrm{HbA}_{1 \mathrm{c}}$ of $\leq 6 \%(42 \mathrm{mmol} / \mathrm{mol})$, with or without diabetes medications. All of those achieving the glycaemic threshold in the LRYGB group did so without diabetes medications, compared to only $72 \%$ of patients in the SG group, so the recalculated proportions for those achieving $\mathrm{HbA}_{1 \mathrm{c}}$ of $\leq 6 \%$ $(42 \mathrm{mmol} / \mathrm{mol})$ without diabetes medication in the two bariatric surgery groups was $42 \%$ after LRYGB and $27 \%$ after SG. In a small study of 41 Israeli patients with T2D $\left(\right.$ BMI $\left.>35 \mathrm{~kg} / \mathrm{m}^{2}\right), 37$ completed 1-year follow-up after randomisation to LRYGB or SG. ${ }^{4}$ There was a similar reduction in $\mathrm{HbA}_{1 \mathrm{c}}$ after LRYGB (by $1.57 \pm 1.35 \%$ or 17 $\pm 15 \mathrm{mmol} / \mathrm{mol}$ ) and LSG (by $2.37 \pm 2.22 \%$ or 26 $\pm 24 \mathrm{mmol} / \mathrm{mol}$ ), $\mathrm{p}=0.34 .{ }^{4}$ In a double-blinded, singlecentre study of 60 Taiwanese patients with T2D (BMI: $\left.25-34 \mathrm{~kg} / \mathrm{m}^{2}\right), 93 \%$ of those randomised to 'mini' bypass achieved diabetes remission at 12 months compared to $47 \%$ randomised to LSG, using diabetes remission criteria of fasting glucose $<7.0 \mathrm{mmol} / \mathrm{L}$ and $\mathrm{HbA}_{1 \mathrm{c}}<6.5 \%(48 \mathrm{mmol} / \mathrm{mol})$ in the absence of diabetes medications. ${ }^{5}$ None of these studies reported their medication adjustment protocol after surgery. The assessment of T2D remission may be affected by participant lifestyle factors and clinician variation in glucose medication withdrawal thresholds used. Further studies evaluating comparative efficacy of LSG and LRYGB are required, particularly using blinding of patients and investigators assessing for T2D remission using standard protocols for postoperative medical management to minimise bias.

The advantages of LRYGB include being fully reversible; however, the irreversible LSG is a faster and simpler procedure with potentially less dumping. There are technical difficulties involved in performing LRYGB in severely obese patients, and such patients may have limited success from LRYGB attributed to pouch dilation and loss of restriction at the gastrojejunal anastomosis over time. The placement of a silastic ring (SR) band around the gastric pouch at the time of primary RYGB is considered superior to the non-banded RYGB in the superobese population. ${ }^{6}$ Other modifications to the LRYGB procedure include variation in pouch size $(10-50 \mathrm{~mL})$, alimentary limb length $(50-250 \mathrm{~cm})$ and biliopancreatic limb length $(25-150 \mathrm{~cm}){ }^{2}$

The underlying mechanisms by which SG and RYGB achieve T2D remission are unclear and may involve changes in gut hormones, ${ }^{7}$ inflammatory markers ${ }^{8}$ and gut microbiota. ${ }^{9}$ Investigation into the impact of these two types of bariatric surgery on these mechanisms and resulting glucose metabolism, body composition and satiety is required.

The primary objective of this trial is to compare the efficacy of SR-LRYGB and LSG on remission of T2D, defined by $\mathrm{HbA}_{1 \mathrm{c}}<6 \%(42 \mathrm{mmol} / \mathrm{mol})$ without the use of diabetes medications (as per the consensus definition of complete diabetes remission ${ }^{10}$ ), at 5 years postsurgery. Secondary objectives are to examine proportions achieving alternative glycaemic thresholds
$\mathrm{HbA}_{1 \mathrm{c}}<5.7 \%(39 \mathrm{mmol} / \mathrm{mol})$ or $<6.5 \%(48 \mathrm{mmol} / \mathrm{mol})$ without the use of diabetes medications, extent of weight loss, change in body composition, resting energy expenditure (REE), operative complications, revision rate, hospitalisations, mortality, microvascular and macrovascular complications, cardiovascular risk factors, quality of life, anxiety and depression scores between the two groups. In addition, underlying mechanisms of T2D remission will be investigated by examining comparative changes in gut hormones, inflammatory markers, gut microbiota, in relation to diabetes remission, changes in body composition, food intake and appetite scores.

\section{METHODS}

\section{Trial design}

This is a parallel (1:1), single-centre, two-arm, randomised, double-blind (patient and assessor), superiority trial (figure 1).

\section{Sample size justification and power calculation}

Assuming rates of diabetes remission to be $88 \%$ in SR-LRYGB and $59 \%$ in LSG, a minimum of 42 patients per arm, will provide $80 \%$ power to detect a difference between the two groups using a two-sided $\alpha$ of 0.05 . These estimates were derived from our unpublished audit data. An expected loss to follow-up rate of $20 \%$ requires at least 53 patients per arm.

\section{Data analysis plan}

Study analysis will be by intention-to-treat. Prior to performing analyses, standard data screening and cleaning procedures will be applied to detect possible data-entry errors and to check for outliers, assess the extent and patterns of missing data and check that appropriate assumptions of normality are met whenever necessary. Baseline characteristics will be analysed by descriptive statistics using means and SDs for all continuous variables with a normal distribution, and medians and IQRs for variables with a non-normal distribution. Categorical variables will be summarised with frequencies. For the primary analysis, the difference in proportions achieving T2D remission $\left(\mathrm{HbA}_{1 \mathrm{c}}<6 \% \quad(42 \mathrm{mmol} / \mathrm{mol})\right.$ without diabetes medication) will be compared between LSG and SR-LRYGB at 5 years, adjusting for stratification variables using logistic regression. A two-sided $p$ value of 0.05 will be considered to indicate statistical significance. Missing data will be handled by multiple imputation as appropriate. Analyses will be performed with the use of SAS software, V.9.4 or later (SAS Institute, Cary, North Carolina, USA).

\section{Participants}

All patients aged 20-55 years with T2D of at least 6 months duration, BMI $35-65 \mathrm{~kg} / \mathrm{m}^{2}$ for at least 5 years, who were referred for consideration of bariatric surgery at a single centre (North Shore Hospital), were invited to participate and to attend a bariatric surgery study information evening. All participants were given a 
written informed consent form and understood that on entering the randomised study, they would not know their treatment allocation until completion of the study at 5 years. Other inclusion criteria included being suitable for either of the two surgical procedures, able to give informed consent and committed to follow-up. Exclusion criteria included postprandial $\mathrm{C}$ peptide $<350 \mathrm{pmol} / \mathrm{L}$, pregnancy, type 1 diabetes or secondary diabetes, chronic pancreatitis, oral steroid therapy, current smokers and those not suitable for general anaesthesia. The study commenced in September 2011 and completed recruitment in October 2014. A total of 114 participants were recruited into the study (figure 1). Data collection and follow-up is ongoing.

\section{Baseline assessments}

All participants were prescribed a very low calorie diet (VLCD) with three servings of Optifast (Nestle, Vevey,

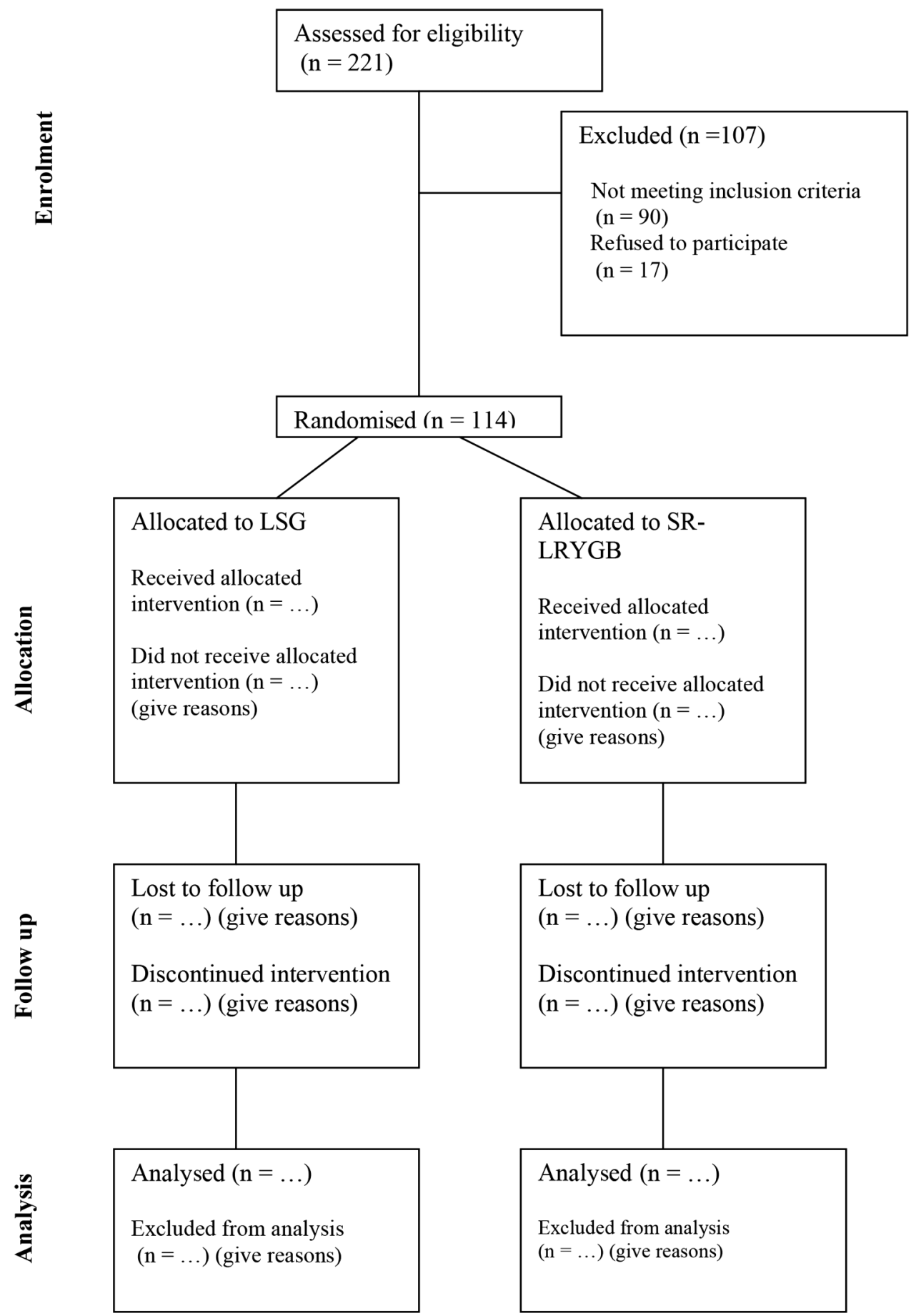

Figure 1 CONSORT diagram showing the proposed flow of participants through the sleeve gastrectomy versus gastric bypass trial for type 2 diabetes. 
Switzerland), each containing $\sim 152$ calories, plus vegetables preoperatively, for 2 weeks, designed to reduce liver fat and make laparoscopic abdominal surgery safer. Baseline clinical and anthropometric assessments were conducted before and after the VLCD. Baseline body composition assessment was conducted during the VLCD period, in the week before surgery (table 1).

\section{Randomisation}

Computer-generated random number codes (Minim, London) managed by an independent study member were used to randomise participants 1:1 to either LSG or SR-LRYGB, stratified by age category (20-29, 30-39 or 40$55)$, BMI category (35-44.9, 45-54.9 and 55-65 kg/m²), ethnicity (Maori, Pacific, NZ European/other), duration of diabetes diagnosis $(<5,5-10$ and $>10$ years $)$ and the presence of insulin therapy.

\section{Allocation concealment and blinding}

On the day of surgery, following induction of general anaesthesia, allocation to either LSG or SR-LRYGB was disclosed only to the operating surgical team. Both operations were performed using identical incisions with a four-port technique (optical entry; two 10-12 mm ports and two $5 \mathrm{~mm}$ ports) and an additional epigastric incision for liver retraction. Participants and all other research and clinical team members remain blinded to surgical allocation. Only de-identified codes were used to link participants to their data during the study to maintain their confidentiality.

\section{Intervention}

For $\mathrm{SG}$, a sleeve was fashioned starting $2 \mathrm{~cm}$ proximal to the pylorus using serial applications of an Echelon Flex 45 stapler (Ethicon) over a 36Fr oro-gastric bougie. For SR-LRYGB, a lesser curve-based gastric pouch was fashioned over a 32Fr oro-gastric tube, with a $50 \mathrm{~cm}$ biliopancreatic limb, $100 \mathrm{~cm}$ antecolic Roux limb with hand-sewn single layer gastrojejunostomy over a 32Fr oro-gastric tube. A $6.5 \mathrm{~cm}$ SR was then secured around the gastric pouch $2 \mathrm{~cm}$ above the gastrojejunostomy anastomosis. Mesenteric defects were closed.

\section{Follow-up}

Postoperative care and follow-up will be identical for both groups. All pharmacological agents for diabetes and hypertension will be stopped at the time of surgery. Glucose-lowering therapy will be restarted if mean postoperative capillary glucose exceeds $12 \mathrm{mmol} / \mathrm{L}$. All participants will be reviewed by an endocrinologist at 6 weeks, 9 months then annually (table 1) for adjustment of all medications and assessment of microvascular and macrovascular complications. ${ }^{11}$ The medication adjustment protocol including lipid, blood pressure and glucose-lowering therapy is outlined in figure 2. Microvascular complications will be assessed annually with clinical evaluation for peripheral neuropathy symptoms and signs, retinal photoscreening, measurement of renal function and urine albumin:creatinine ratio. Macrovascular complications such as incidence of myocardial infarction, stroke and peripheral vascular disease will also be recorded.

\section{Assessment of outcomes}

$\mathrm{HbA}_{1 \mathrm{c}}$ will be measured by high-performance liquid chromatography (Bio-Rad). Body weight will be recorded to the nearest $0.1 \mathrm{~kg}$ using digital scales (SECA, Chino, California, USA). Height will be recorded to the nearest $0.5 \mathrm{~cm}$ using a stadiometer. Total body fat, left femoral neck bone density and anteroposterior (AP) lumbar spine bone density will be measured by dual-energy X-ray absorptiometry (model iDXA, software V.15, GE-Lunar, Madison, Wisconsin, USA). Per cent body fat will be calculated as $100 \times$ total body fat/body weight. REE will be measured after overnight fast using a ventilated canopy connected to an open-circuit indirect calorimeter (Deltatrac Metabolic Monitor MBM-100, Datex Instruments, Helsinki, Finland). Hospitalisations, operative complications graded according to the Clavien-Dindo classification, ${ }^{12}$ mortality, revisional surgery and changes in medications will be recorded. Hospital anxiety and depression scale ${ }^{13}$ and 36 -item short form ${ }^{14}$ questionnaires will be used (table 1).

\section{Ancillary mechanistic study}

Alongside the primary trial, participants were able to opt in to an exploratory gut hormone and gut bacteria mechanistic substudy. As part of this study, they were asked to provide additional data and biosamples during the three scheduled visits for body composition assessments at baseline, 1 and 5 years. The additional data include a 3-day food diary, hunger ratings assessment, fecal samples and a $75 \mathrm{~g}$ oral glucose tolerance test. Participants were requested to prospectively record all foods and drinks taken during the 3-day diary period, including the amounts taken, any dietary supplements taken and medications taken during the period. Visual analogue scale hunger ratings will be collected on arrival at the body composition unit in a fasted state at baseline, 1 and 5 years. Participants will be asked to rate their motivation to eat on a horizontal non-graded line measuring $100 \mathrm{~mm}$, anchored on the left by 'not at all' and on the right by 'very much' next to four responses: How hungry are you? How full do you feel? How strong is your desire to eat? How much food do you think you could eat? Fecal samples will be self-collected in stool containers, sealed and placed into another sealed container filled with water and frozen immediately at $-20^{\circ}$ C, before being transported in the frozen state to the laboratory where they will be stored at $-80^{\circ} \mathrm{C}$. Participants will be asked to attend these body composition/REE visits in a fasted state for a 2-hour $75 \mathrm{~g}$ oral glucose tolerance test, with $30 \mathrm{~min}$ blood sampling. Blood samples will be collected into EDTA, serum separator tubes and BD P800 tubes (BD, Franklin Lakes, New Jersey, USA), containing 
Table 1 Study timeline and investigations

\begin{tabular}{|c|c|c|c|c|c|c|c|c|c|c|c|c|}
\hline & Baseline & Week 1 & Week 6 & 3 months & 6 months & 9 months & 12 months & 18 months & 2 years & 3 years & 4 years & 5 years \\
\hline $\begin{array}{l}\text { Clinical history and } \\
\text { medications }\end{array}$ & $\checkmark$ & $\checkmark$ & $\checkmark$ & $\checkmark$ & $\checkmark$ & $\checkmark$ & $\checkmark$ & $\checkmark$ & $\checkmark$ & $\checkmark$ & $\checkmark$ & $\checkmark$ \\
\hline Blood pressure & $\checkmark$ & $\checkmark$ & $\checkmark$ & $\checkmark$ & $\checkmark$ & $\checkmark$ & $\checkmark$ & $\checkmark$ & $\checkmark$ & $\checkmark$ & $\checkmark$ & $\checkmark$ \\
\hline Anthropometrics & $\checkmark$ & $\checkmark$ & $\checkmark$ & $\checkmark$ & $\checkmark$ & $\checkmark$ & $\checkmark$ & $\checkmark$ & $\checkmark$ & $\checkmark$ & $\checkmark$ & $\checkmark$ \\
\hline DEXA/REE* ${ }^{\star}$ & $\checkmark$ & & & & & & $\checkmark$ & & & & & $\checkmark$ \\
\hline Endocrinology review & 1 & & $\checkmark$ & & & $d$ & & 21 months & & 33 months & 45 months & 57 months \\
\hline $\begin{array}{l}\text { Hospital anxiety and } \\
\text { depression score }\end{array}$ & $\checkmark$ & & $\checkmark$ & $\checkmark$ & $\checkmark$ & $\checkmark$ & $\checkmark$ & $\checkmark$ & $\checkmark$ & $\checkmark$ & $\checkmark$ & $\checkmark$ \\
\hline $\begin{array}{l}\text { Short form health survey } \\
\text { instrument (SF-36) }\end{array}$ & $\checkmark$ & & $\checkmark$ & $\checkmark$ & $\checkmark$ & $\checkmark$ & $\checkmark$ & $\checkmark$ & $\checkmark$ & $\checkmark$ & $\checkmark$ & $\checkmark$ \\
\hline Laboratory tests $\dagger$ & $\checkmark$ & $\checkmark$ & $\checkmark$ & $\checkmark$ & $\checkmark$ & $\checkmark$ & $\checkmark$ & $\checkmark$ & $\checkmark$ & $\checkmark$ & $\checkmark$ & $\checkmark$ \\
\hline $\mathrm{HbA}_{1 \mathrm{c}}$ & $\checkmark$ & & & $\checkmark$ & $\checkmark$ & $\checkmark$ & $\checkmark$ & $\checkmark$ & $\checkmark$ & $\checkmark$ & $\checkmark$ & $\checkmark$ \\
\hline $\begin{array}{l}\text { Stored fasting plasma } \\
\text { and serum }\end{array}$ & $\checkmark$ & $\checkmark$ & $\checkmark$ & $\checkmark$ & $\checkmark$ & $\checkmark$ & $\checkmark$ & $\checkmark$ & $\checkmark$ & $\checkmark$ & $\checkmark$ & $\checkmark$ \\
\hline \multicolumn{13}{|l|}{ Mechanistic substudy } \\
\hline Food diary & $\checkmark$ & & & & & & $\checkmark$ & & & & & $\checkmark$ \\
\hline Satiety questionnaire & $\checkmark$ & & & & & & $\checkmark$ & & & & & $\checkmark$ \\
\hline Fecal sample $\ddagger$ & 1 & & & & & & 1 & & & & & 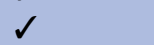 \\
\hline $\begin{array}{l}\text { Plasma and serum } \\
\text { samples from oral } \\
\text { glucose tolerance test } \$\end{array}$ & 1 & & & & & & $\checkmark$ & & & & & $\checkmark$ \\
\hline
\end{tabular}

${ }^{*}$ Dual-energy X-ray absorptiometry/resting energy expenditure.

†Full blood count, C reactive protein, ESR, electrolytes, creatinine, calcium, albumin, bilirubin, liver enzymes, lipids and 25-hydroxy-vitamin D.

†Samples immediately frozen.

$\S$ Samples also stored in BD P800 tubes for gut hormone analysis. 
While inpatient at the time of surgery:

- Stop all diabetes, hypertension and lipid lowering therapies (and aspirin) at the time of surgery. Exceptions to this are:

* In those where aspirin and/or lipid lowering therapy is being used for secondary prevention (previous cardiovascular events) - aspirin/ lipid lowering treatment should not be stopped

- $\quad$ * In those with microalbuminuria - Angiotensin Convertase Esterase-inhibitor (ACEI) /Angiotensin Receptor Blocker (ARB) therapy should not be stopped

- Diabetologist to review all trial patients prior to discharge and:

* Restart antihypertensive therapy in those with post-op mean BP $>150 / 90 \mathrm{mmHg}$

* Restart diabetes treatment in those with post-operative mean capillary glucose $>12$ $\mathrm{mmol} / \mathrm{L}$

(Regimen of antihypertensive and/or diabetes to be decided by the diabetologist reflecting pre-operative treatment, and likely strength of therapy required)

\section{During follow-up visits within the 5 year trial period:}

- Those who are still on any therapy for diabetes, hypertension or microalbuminuria:

* Stop/wean diabetes treatment if the latest HbAlc is $<53 \mathrm{mmol} / \mathrm{mol}$

* Stop/wean antihypertensive if BP $<140 / 90$ (repeat BP +/- 24 hour ambulatory BP monitoring if in doubt)

* Stop ACE-inhibitor/ARB if latest urinary microalbumin level normal

* Stop/wean statin/lipid lowering therapy (unless this is for secondary prevention) if 5 year cardiovascular risk has fallen below $15 \%$ using New Zealand Society for Study of Diabetes CVD risk calculator (nzssd.org.nz/cvd) [9]

- Initiate or augment medical therapy in the following situations:

*CVD event (CAD/ CVA) mandating appropriate therapy (anti-platelet/aspirin, lipid lowering, $\mathrm{BP}$ lowering treatments)

* 2 x latest consecutive $\mathrm{HbA} 1 \mathrm{c}$ of $53 \mathrm{mmol} / \mathrm{mol}$ or above - start diabetes treatment (metformin in almost all instances )

- $\quad *$ Blood pressure $>140 / 90$ (repeat if $1 \mathrm{x}$ raised, consider $24 \mathrm{hr}$ ambulatory BP monitoring) - start BP lowering therapy (an ACE-inhibitor in almost all instances) *Newly positive urinary microalbumins - start an ACE-inhibitor (ARB if intolerant) *5 year CVD risk $>15 \%$ using NZSSD CVD risk calculator - start lipid lowering therapy (a statin in almost all instances)

Figure 2 Endocrinology evaluation and treatment protocol for trial patients.

protease inhibitors to maximise the stability of gut hormones. $^{15}$

\section{Ethics and dissemination}

Ethics approval has been granted by the New Zealand regional ethics committee (NZ93405). This study was prospectively registered at ANZCTR (ACTRN12611000751976) and retrospectively registered at clinicaltrials.gov (NCT01486680). The results of this study and ancillary studies will be publicised in the form of presentations at national and international meetings. The study and conclusions regarding the primary and secondary objectives and ancillary studies will be presented as manuscripts submitted for peer-reviewed journal publication.

\section{DISCUSSION}

This is the first double-blind, randomised trial to compare SR-LRYGB and LSG for the treatment of T2D in morbidly obese patients including those with BMI up to $65 \mathrm{~kg} / \mathrm{m}^{2}$. The use of a standard metabolic medication adjustment protocol is a strength of the study design, in effort to reduce heterogeneity in management of blood pressure, lipids and T2D postoperatively. The ancillary studies interrogating comparative changes in gut microbiota and gut hormones may uncover novel mechanistic insights into how diabetes remission is achieved through these two contrasting surgical procedures.

The term 'remission' with 'partial' and 'complete' descriptors has been used within the bariatric surgery 
literature with distinct thresholds of $\mathrm{HbA}_{1 \mathrm{c}}$ and fasting glucose, generally in the absence of glucose lowering therapy, to represent varying degrees of diabetes improvement. ${ }^{10}$ However, these are controversial given that the diagnosis of diabetes itself is not dichotomous and rather thresholds of glycaemia have been defined on the basis of the associated risk of microvascular and macrovascular complications. It is not yet known whether these thresholds remain true in a postbariatric surgery population, and consequently, diagnostic criteria for prediabetes and diabetes validated for a non-surgical population may be misleading when applied in reverse, to those who have undergone bariatric surgery. Similarly, there is a paucity of evidence to guide the development of valid and reliable protocols for discontinuing cardiovascular risk-modifying medications after bariatric surgery for optimum medical management. Nonetheless, we have selected one of the most commonly accepted $\mathrm{HbA}_{1 \mathrm{c}}$ thresholds for classifying diabetes remission ${ }^{16}$ and used a standard medical management to reduce complacency in medical therapy after abrupt withdrawal of medications postoperatively.

Limitations of this study include the single-centre design and the relatively small sample size. However, by employing stratification for confounding variables in randomisation, this will ensure that these factors (such as duration of T2D, insulin use, ethnicity and age) will be matched across both treatment groups. SR-LRYGB was chosen due to superior long-term weight loss outcomes, largely due to reduction in weight regain when compared to non-banded LRYGB. ${ }^{17-19}$ However, this modification of LRYGB is possibly not widely adopted due to unfamiliarity with placing it and potential issues regarding food intolerance and band-related complications. ${ }^{6}$ Some of these concerns are ill conceived and hence currently the use of SR type of LRYGB may limit generalisability of the study.

\section{CONCLUSION}

This article presents the protocol and data analysis plan for a single-centre, randomised, double-blinded clinical study comparing LSG and SR-LRYGB in the treatment of T2D in morbidly obese patients, including those who are super-obese. The results of this study, when completed, will assist in decision-making between LSG and LRYGB for the treatment of T2D in morbidly obese patients. In the interim, we hope this description of the study design and metabolic medication adjustment protocol will assist clinicians looking after patients following bariatric surgery and researchers in planning future bariatric surgery trials.

\footnotetext{
Author affiliations

${ }^{1}$ Faculty of Medical and Health Sciences, Department of Medicine, University of Auckland, Auckland, New Zealand

${ }^{2}$ Department of Surgery, North Shore Hospital, Waitemata District Health Board, Auckland, New Zealand

${ }^{3}$ Department of Endocrinology, North Shore Hospital, Waitemata District Health Board, Auckland, New Zealand
}

${ }^{4}$ Faculty of Medical and Health Sciences, Department of Surgery, University of Auckland, Auckland, New Zealand

Contributors MWCB and RM conceived this study. HH, LDP, RC, DDK, SJR, MGC, NJE and LH contributed to study design. MWCB, HH, NJE and SJR were primarily responsible for the surgical aspects of the protocol. RC, DDK and RM were primarily responsible for the medical assessment protocol of participants. LDP is primarily responsible for the body composition assessment and energy expenditure protocol. RM is primarily responsible for the gut microbiota and gut hormone substudy protocol. RM wrote the first draft of this manuscript. All authors read and contributed to the final draft of the paper.

Funding We acknowledge the funding support from Waitemata District Health Board, which provides limited publically funded bariatric surgery ( $\sim 100$ cases annually). Additional funding for blood sample storage and a research nurse salary was provided by Johnson and Johnson (NZ), Covidien (NZ) and Obex (NZ). DEXA scanning for body composition was provided by the Diabetes Research Fund (NZ) and Maurice \& Phyllis Paykel Trust (NZ). Sample collection for the gut microbiota and gut hormone substudy was funded by a grant from the Maurice Wilkins Centre, New Zealand. None of these funders had any role in study design or data analysis or interpretation.

Competing interests None declared.

Patient consent Obtained.

Ethics approval New Zealand regional ethics committee.

Provenance and peer review Not commissioned; externally peer reviewed.

Data sharing statement All enquiries regarding data from this study should be made to the corresponding author.

Open Access This is an Open Access article distributed in accordance with the Creative Commons Attribution Non Commercial (CC BY-NC 4.0) license, which permits others to distribute, remix, adapt, build upon this work noncommercially, and license their derivative works on different terms, provided the original work is properly cited and the use is non-commercial. See: http:// creativecommons.org/licenses/by-nc/4.0/

\section{REFERENCES}

1. Yip S, Plank LD, Murphy R. Gastric bypass and sleeve gastrectomy for type 2 diabetes: a systematic review and meta-analysis of outcomes. Obes Surg 2013;23:1994-2003.

2. Li J, Lai D, Wu D. Laparoscopic Roux-en-Y gastric bypass versus laparoscopic sleeve gastrectomy to treat morbid obesity-related comorbidities: a systematic review and meta-analysis. Obes Surg 2016;26:429-42.

3. Schauer PR, Kashyap SR, Wolski K, et al. Bariatric surgery versus intensive medical therapy in obese patients with diabetes. $N$ Engl J Med 2012;366:1567-76.

4. Keidar A, Hershkop KJ, Marko L, et al. Roux-en-Y gastric bypass vs sleeve gastrectomy for obese patients with type 2 diabetes: a randomised trial. Diabetologia 2013;56:1914-18.

5. Lee WJ, Chong K, Ser KH, et al. Gastric bypass vs sleeve gastrectomy for type 2 diabetes mellitus: a randomized controlled trial. Arch Surg 2011;146:143-8.

6. Mahawar KK, Parikh C, Carr WR, et al. Primary banded Roux-en-Y gastric bypass: a systematic review. Obes Surg 2014;24:1771-92.

7. Vetter ML, Cardillo S, Rickels MR, et al. Narrative review: effect of bariatric surgery on type 2 diabetes mellitus. Ann Intern Med 2009;150:94-103.

8. Iannelli A, Anty R, Piche T, et al. Impact of laparoscopic Roux-en-Y gastric bypass on metabolic syndrome, inflammation, and insulin resistance in super versus morbidly obese women. Obes Surg 2009;19:577-82.

9. Aron-Wisnewsky J, Dore J, Clement K. The importance of the gut microbiota after bariatric surgery. Nat Rev Gastroenterol Hepatol 2012;9:590-8.

10. Buse JB, Caprio S, Cefalu WT, et al. How do we define cure of diabetes? Diabetes Care 2009;32:2133-5.

11. Elley CR, Robinson E, Kenealy $\mathrm{T}$, et al. Derivation and validation of a new cardiovascular risk score for people with type 2 diabetes: the New Zealand diabetes cohort study. Diabetes Care 2010;33: 1347-52. 
12. Dindo D, Demartines N, Clavien PA. Classification of surgical complications: a new proposal with evaluation in a cohort of 6336 patients and results of a survey. Ann Surg 2004;240:205-13.

13. Bjelland I, Dahl AA, Haug TT, et al. The validity of the Hospital Anxiety and Depression Scale. An updated literature review. $J$ Psychosom Res 2002;52:69-77.

14. Ware JE Jr, Sherbourne CD. The MOS 36-item short-form health survey (SF-36). I. Conceptual framework and item selection. Med Care 1992;30:473-83.

15. Yi J, Warunek D, Craft D. Degradation and stabilization of peptide hormones in human blood specimens. PLoS One 2015;10: e0134427.
16. Miras AD, Risstad H, Baqai N, et al. Application of the international diabetes federation and American diabetes association criteria in the assessment of metabolic control after bariatric surgery. Diabetes Obes Metab 2014;16:86-9.

17. Bessler M, Daud A, Kim T, et al. Prospective randomized trial of banded versus nonbanded gastric bypass for the super obese: early results. Surg Obes Relat Dis 2007;3:480-4.

18. Awad W, Garay A, Martinez C. Ten years experience of banded gastric bypass: does it make a difference? Obes Surg 2012;22:271-8.

19. Heneghan HM, Annaberdyev S, Eldar S, et al. Banded Roux-en-Y gastric bypass for the treatment of morbid obesity. Surg Obes Relat Dis 2014;10:210-16. 